\title{
Time-synchronized immune-guided SBRT partial bulky tumor irradiation targeting hypoxic segment while sparing the peritumoral immune microenvironment
}

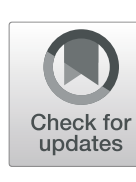

Slavisa Tubin ${ }^{1 *}$ (D), Martin Ashdown ${ }^{2}$ and Branislav Jeremic ${ }^{3}$

\begin{abstract}
Background: A novel unconventional SBRT-based PArtial Tumor irradiation targeting HYpoxic clonogenic cells (SBRT-PATHY) for induction of the tumoricidal bystander (BE) and abscopal effects (AE) was developed by translating our preclinical findings to a clinic in 2016. In order to further improve BE/AE response rate, SBRT-PATHY was upgraded in 2018 by the sparing of peritumoral immune microenvironment as a new OAR, defined by its own dose-constraints. Considering the anti-tumor immune response homeostatic fluctuation, which is cyclically suppressed and incited ("switched off and on"), we synchronized SBRT-PATHY with its most excitable phase, in order to overcome tumor tolerance locally and systemically. The aim of this study, therefore, was to report on the initial results of our latest innovation aimed to further improve BE/AE response rate by testing the effectiveness of the time-synchronized immune-guided SBRT-PATHY.
\end{abstract}

Materials and methods: In order to serially map the homeostatic anti-tumor immune response-fluctuations, High Sensitive C-Reactive Protein (HS-CRP), Lactate Dehydrogenase (LDH) and Lymphocyte/Monocyte Ratio (LMR) were analyzed using high-order polynomial trend analysis as surrogate of immune system response. After the biomarker data analysis detected the immune fluctuations and related idiosyncratic immune cycle periodicity, we determined the "most favourable" and "least favourable" treatment time-positions in the immune cycle. In order to evaluate the impact of an idiosyncratic immune cycle on treatment outcomes, our first consecutive four patients were treated on the "most favourable" while the remaining four on the "least favourable" day.

Results: The median follow-up was 11.8 months. The biomarker data analysis showed periodic immune response fluctuations of regular frequency. The "right" synchronization of SBRT-PATHY with the "most favorable day" of antitumor immune response was accompanied with improved clinical outcomes in terms of BE/AE-response rate.

Conclusion: We believe the right synchronization of radiotherapy with the homeostatically oscillating immune response may improve the probability of inducing BE/AE.

Present study has been retrospectively registered on 18th of October 2019 by the ethic committee for Austrian region „Kärnten "in Klagenfurt (AUT), under study number A 37/19.

Keywords: Immune-guided timing, Partial irradiation, Bystander effect, Abscopal effect, Tumor hypoxia, Immune microenvironment

\footnotetext{
* Correspondence: slavisa.tubin@kabeg.at

'KABEG Klinikum Klagenfurt, Institute of Radiation Oncology, Feschnigstraße

11, 9020 Klagenfurt am Wörthersee, Austria

Full list of author information is available at the end of the article
}

(c) The Author(s). 2019 Open Access This article is distributed under the terms of the Creative Commons Attribution 4.0 International License (http://creativecommons.org/licenses/by/4.0/), which permits unrestricted use, distribution, and reproduction in any medium, provided you give appropriate credit to the original author(s) and the source, provide a link to the Creative Commons license, and indicate if changes were made. The Creative Commons Public Domain Dedication waiver (http://creativecommons.org/publicdomain/zero/1.0/) applies to the data made available in this article, unless otherwise stated. 


\section{Introduction}

Clinical exploitation of the bystander (BE) and abscopal effects (AE) was an objective of our long-standing translational oncology research aimed to overcome the outcome-limiting factors related to the unresectable bulky tumors. Despite the developments in oncological therapy BE/AE remain still rare phenomena [1].

In order to improve the therapeutic-ratio by exploiting $\mathrm{BE} / \mathrm{AE}$ an unconventional partial tumor irradiation targeting the hypoxic segment was developed in 2016 in our institute [2]. Our preclinical findings indicated that the hypoxic in respect to normoxic tumor cells, if selectively irradiated as inductor of $\mathrm{BE} / \mathrm{AE}$, show higher potential for the generation of BE/AE [3]. The subsequent translation of these findings to a clinic led to the introduction of a novel SBRT-based PArtial Tumor irradiation targeting HYpoxic clonogenic cells (SBRT-PATHY) showing promising BE/AE-response rates $[2,4]$. Recently, the Italian group confirmed efficacy of SBRT-PATHY in their initial experience [5]. Considering the immune-mediated nature of $\mathrm{BE} / \mathrm{AE}$ and in order to further improve $\mathrm{BE} / \mathrm{AE}$ response rate, SBRT-PATHY was upgraded in 2018 by the sparing of peritumoral immune microenvironment as a new OAR, defined by its own dose-constraints $[4,6]$. Our concept implied that for successful therapeutic immune modulation, the entire tumor volume may not need to be irradiated but only a part of the tumor. This should initiate antigen shedding, increase effector $\mathrm{T}$ cell activation and lead to favorable alterations in radiation-spared peritumoral immune environment [7].

Currently, some studies have described an association between the radiation-induced lymphopenia with poor oncologic outcome, indicating that radiotherapy using large volumes and multiple daily fractions can lead to immunosuppression $[8,9]$. On the other side, some studies have shown potential therapeutic benefits by eventual ablation of regulatory ("suppressor") T cells with limited (single-dose) systemic therapies [10-12] given "at the right time" in order to selectively ablate those suppressor $\mathrm{T}$ cells while sparing the effector T cells. Thus, suggesting that the accurate timing of limited therapy may play a major role in treatment efficacy. Following the recent reports of the anti-tumor immune response oscillating over several days [13-16], we hypothesized the following: by monitoring before the treatment immune-specific biomarkers as the surrogates of homeostatically fluctuating immune response, which is cyclically suppressed and incited ("switched off and on"), it would be possible to determine a periodicity of immune response and, based on that, to synchronize SBRT-PATHY with its most excitable phase, in order to overcome tumor tolerance locally and systemically.

The objective of this study, therefore, was to report on the initial results of our latest innovation aimed to further improve $\mathrm{BE} / \mathrm{AE}$ response rate by testing the effectiveness of the time-synchronized immune-guided SBRT-PATHY.

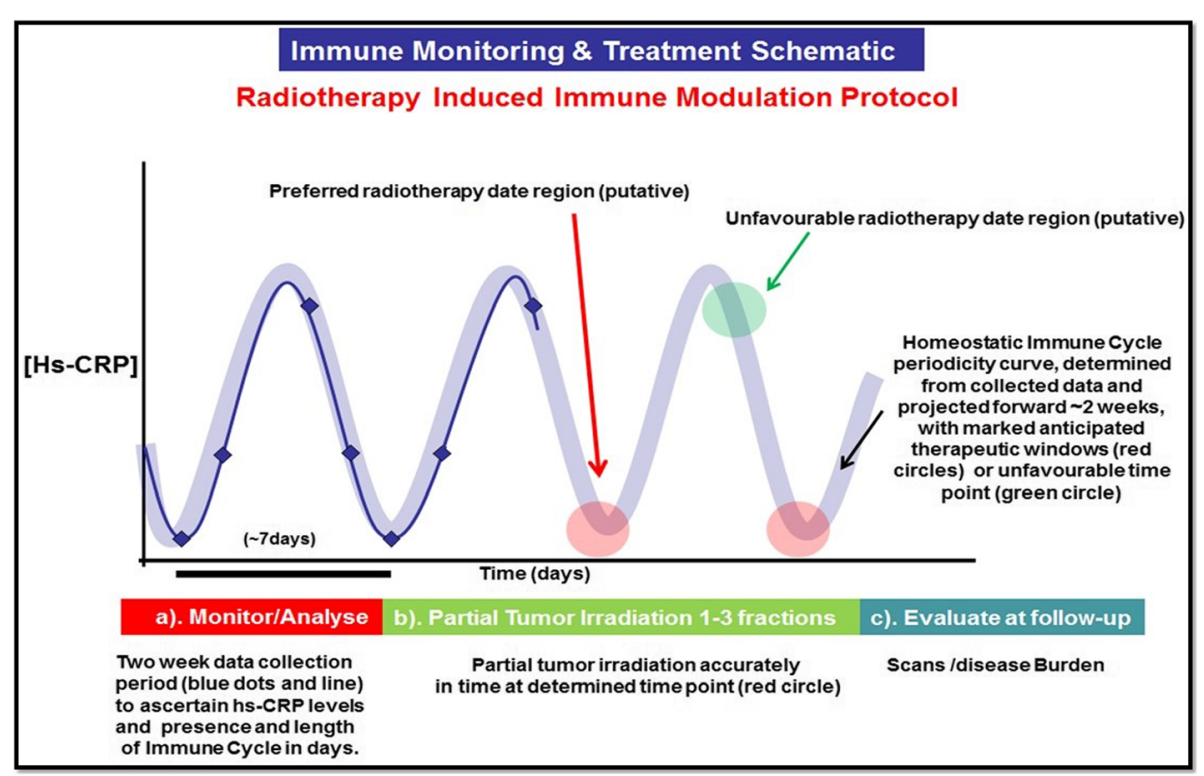

Fig. 1 Radiotherapy induced immune modulation protocol schematic: this figure describes the three phases of the timing of SBRT-PATHY protocol as: (a) the monitoring, collection and analysis of serial data to establish the presence of immune cycle and its periodicity (blue dots and line), (b) projection forward to the putative "most favorable time-position in a cycle" (red circles) or "less favorable time-position"(green circle) to initiate radiotherapy, (c) evaluate at follow-up via scans to provide the evidence for patient responses stratification determined by timing of the treatment 


\section{Materials and methods}

Timing of SBRT-PATHY with respect to an underlying fluctuating anti-tumor immune response

Two weeks prior to initiation of SBRT-PATHY, serial (7x) bloods were taken from each patient every second day and assayed for serum biomarkers such as High Sensitive C-Reactive Protein (HS-CRP), Lactate Dehydrogenase (LDH) and also Lymphocyte/Monocyte Ratio
(LMR). Data from the assays were analysed to define cyclical fluctuations using high-order polynomial trend analysis. In order to determine each patient's idiosyncratic immune cycle periodicity, the trend/periodicity analysis was performed to generate a standard sine wave of similar periodicity (over several cycles) and visually overlayed and aligned (peak \& trough) on the generated polynomial trend graph. This alignment was done in
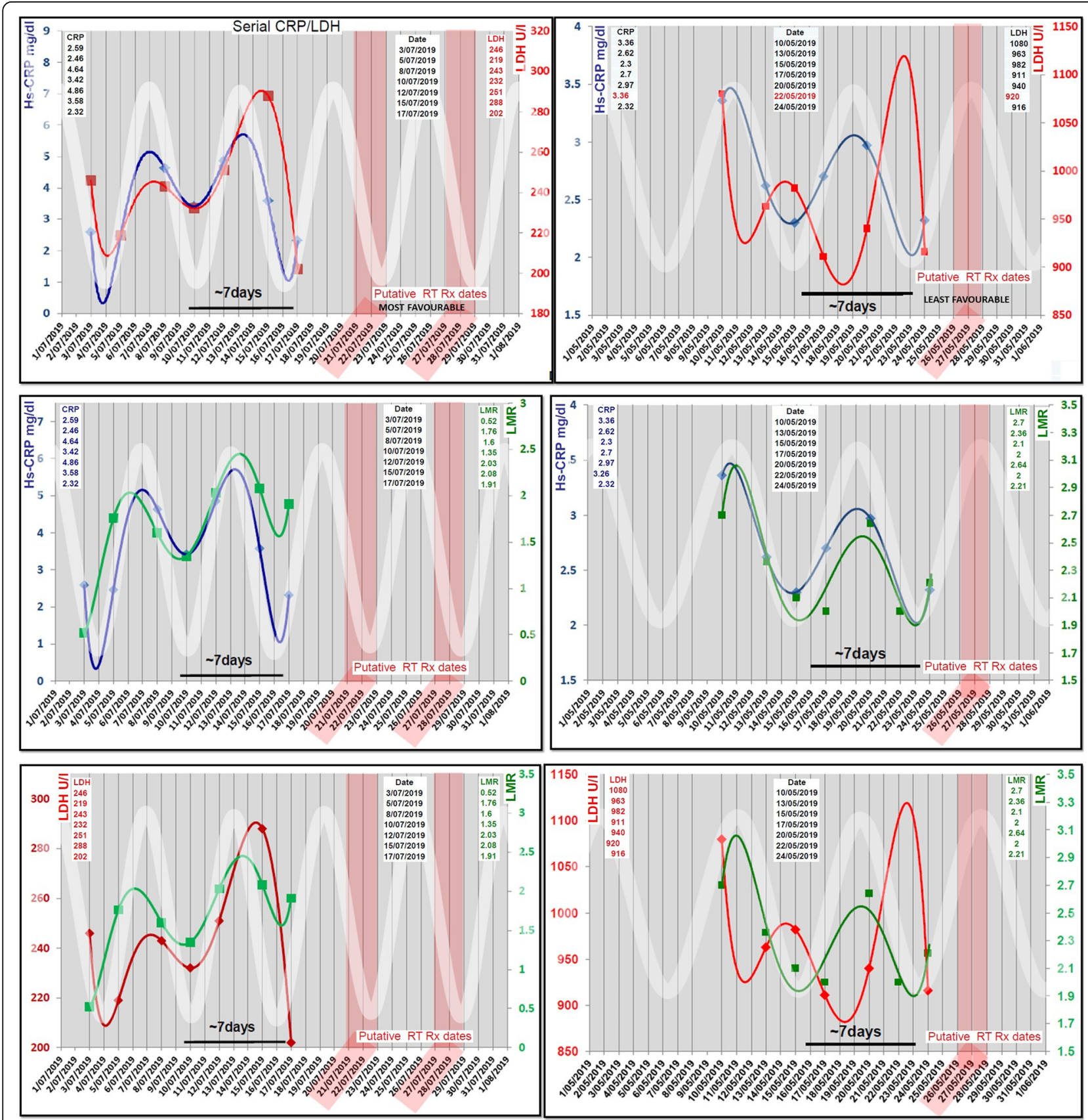

Fig. 2 Immune oscillation monitoring and treatment profile: This figure describes the graphical representation of serial monitoring data over 2 weeks of two example patients in left and right columns. Each graph panel compares the polynomial cyclical relationship and periodicity of biomarkers Hs-CRP/ LDH, Hs-CRP/LMR and LMR/LDH against a standard sine-wave (thick white line). This white sinusoidal line projects forward in time in attempt to identify the putative "most favourable" or "least favourable" dates to treat (marked as red boxes) 
order to project forward in time to the designated/putative treatment date(s) (Figs. 1 and 2). After the biomarker data analysis detected the immune fluctuations and related idiosyncratic immune cycle periodicity, we determined the "most favourable" and "least favourable" treatment time-positions in the immune cycle. As the biomarkers we used are known to rise and fall periodically over several days with the initiation and then homeostatic termination of the immune response $[13,17]$, we defined a pre-trough time-position as the "most favourable" treatment phase in the immune cycle, while a pre-peak region as the "least favourable" day for SBRT-PATHY. In order to evaluate the impact of an idiosyncratic immune cycle on treatment outcomes, our first consecutive four patients were treated on the "most favourable" while the remaining four on the "least favourable" day.

SBRT-PATHY target definition and radiotherapy technique have been previously described in detail [7]. We used a combination of CT and 18F-FDG-PET to define the hypovascularized (contrast-hypo-enhanced) and hypometabolic (SUV max $<3$ ) tumor region representing the "hypoxic" segment between the centralnecrotic and the remaining peripheral-vascularized tumor segments, which was then irradiated with $10 \mathrm{~Gy} \times 3$ to the 70\%-isodose line (Fig. 3).

\section{Patients}

Eight patients with symptomatic, unresectable bulky tumors were prospectively treated between November
2017 and July 2019 with time-synchronized immuneguided SBRT-PATHY. All patients presented either distant metastases or regional metastatic lymph nodes that were not irradiated but followed for AE-induction. The treated patients' main characteristics are summarized in Table 1. Two of eight patients previously received chemotherapy and immunotherapy, and both developed disease progression prior to SBRT-PATHY. No patient received any systemic treatment concomitant with SBRT-PATHY or before the second follow up after it. Two patients which previously received systemic treatment, continued with their treatment after 2 months post-SBRT-PATHY. The response evaluation was performed following the RECIST criteria at 1 month after the treatment by using CT and/or PET-CT, followed by repeated scans at month 2 and then every 3 months. Toxicity was evaluated using the CTCAE Criteria v5.0. All procedures performed in the present study were in accordance with the ethical standards. All the patients signed the informed consent. Present study has been registered by the local ethic committee under study number A 37/19.

\section{Results}

Idiosyncratic immune cycle periodicity

The biomarker data analysis showed immune response fluctuations (Fig. 2) which were synchronized, following similar, regular frequency. The mean immune cycle

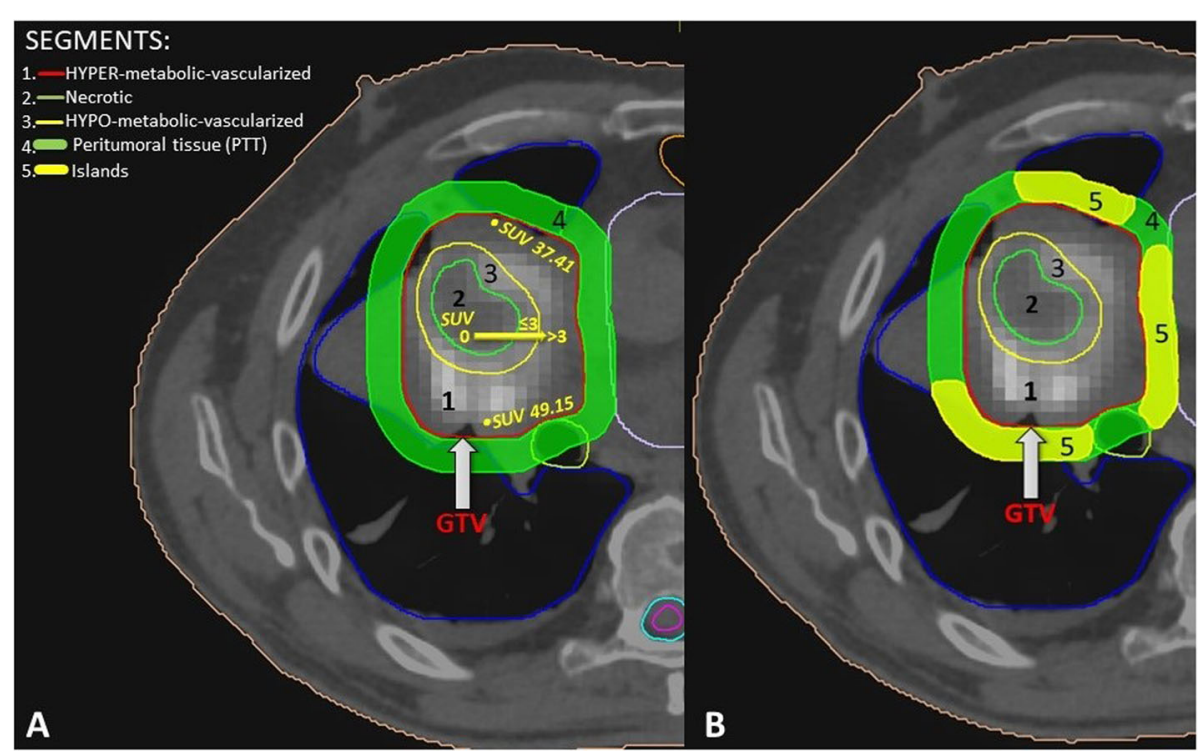

Fig. 3 Target definition by segmentation of an unresectable bulky lung cancer (a): Segment 1- representing the contrast-enhanced (vascularized, "normoxic") peripheral tumor segment, (GTV), Segment 2 - being the contrast-unenhanced (necrotic, "anoxic") central tumor region, Segment 3delineating the contrast-hypo-enhanced (hypovascularized, "hypoxic") junctional tumor region as an up to a maximum of 5 mm junctional zone between the central-necrotic and the remaining peripheral-vascularized tumor segments, Segment 4 - representing peritumoral tissue; (b) Sparing of the peritumoral-surrounding immune microenvironment (thick green line) by creating as an organ at risk multiple "immune microenvironmental islands" (thick yellow line) in order to spare from radiation dose a significant volume (for ex. 2/3) of the tumor microenvironment, leaving it intact and functional 
Table 1 Patient and disease characteristics

\begin{tabular}{|c|c|}
\hline FEATURES: & $\begin{array}{l}\text { Number of } \\
\text { patients } \\
\text { (total 8): }\end{array}$ \\
\hline \multicolumn{2}{|l|}{ GENDER: } \\
\hline Male & 5 \\
\hline Female & 3 \\
\hline \multicolumn{2}{|l|}{ AGE (years): } \\
\hline Mean & 68.4 \\
\hline Range & $57-80$ \\
\hline \multicolumn{2}{|l|}{ ECOG PERFORMANCE STATUS: } \\
\hline $0-1$ & 6 \\
\hline $2-3$ & 2 \\
\hline \multicolumn{2}{|l|}{ PRIMARY TUMOR SITE: } \\
\hline Skin & 1 \\
\hline Breast & 1 \\
\hline Lung & 6 \\
\hline \multicolumn{2}{|l|}{ HISTOLOGY: } \\
\hline Adenocarcinoma (lung) & 3 \\
\hline Adenocarcinoma (breast) & 1 \\
\hline Squamous (lung) & 3 \\
\hline Malignant melanoma & 1 \\
\hline \multicolumn{2}{|l|}{ TREATED BULKY TUMOR SITE: } \\
\hline Lung primary & 6 \\
\hline Neck lymph node metastasis (skin melanoma) & 1 \\
\hline Bone metastasis (breast) & 1 \\
\hline $\begin{array}{l}\text { BULKY TUMOR PATIENTS WITH DISTANT } \\
\text { OLIGOMETASTASES: }\end{array}$ & 3 \\
\hline \multicolumn{2}{|l|}{$\begin{array}{l}\text { Lung primary(1), malignant melanoma (1), } \\
\text { Breast(1), }\end{array}$} \\
\hline $\begin{array}{l}\text { BULKY TUMOR PATIENTS WITH LYMPH NODE } \\
\text { METASTASES ONLY: }\end{array}$ & 5 \\
\hline \multicolumn{2}{|l|}{ Lung primary(5) } \\
\hline $\begin{array}{l}\text { UNRESCTABLE BULKY TUMOR DIAMETER: } \\
\text { mean/range }(\mathrm{cm})\end{array}$ & $10.1 / 6.6-13.8$ \\
\hline $\begin{array}{l}\text { UNRESCTABLE BULKY TUMOR VOLUME: } \\
\text { mean/range }\left(\mathrm{cm}^{3}\right)\end{array}$ & $375.4 / 121.5-901.8$ \\
\hline $\begin{array}{l}\text { TARGETED BULKY TUMOR HYPOXIC SEGMENT: } \\
\text { mean/range }\left(\mathrm{cm}^{3}\right)\end{array}$ & $124.7 / 41.9-442.9$ \\
\hline \multicolumn{2}{|l|}{ SYSTEMIC THERAPY (exclusively before SBRT-PATHY) } \\
\hline Chemotherapy & 1 \\
\hline Immunotherapy & 1 \\
\hline \multicolumn{2}{|l|}{ SYMPTOMS related to bulky disease: } \\
\hline Pain & 6 \\
\hline Dyspnea & 7 \\
\hline Cough & 5 \\
\hline
\end{tabular}

FEATURES:

duration was 7.3 days (range: 6.5-10). The average HS$\mathrm{CRP}$ and LDH concentrations were $2.92 \mathrm{mg} / \mathrm{dl}$ (range: 0.13-7.35) and 492.8 (range: 163-1080), respectively.

\section{Clinical outcomes}

The median follow-up was 11.8 months (range: 4-22). At the time of analysis, one patient (treated at "most favourable" time-position) had died 22 months after SBRTPATHY because of causes other than cancer. A significant $\mathrm{BE}$ (defined as a $30 \%$ or greater regression of partially treated bulky tumor) was observed in all four patients treated at "most favourable" time-position (including those two previously being in progression under systemic treatment) with an average tumor shrinkage of $100 \%$ (three complete responses (CR), one $80 \%$ tumor regression), while among those treated at "least favourable" time-position in two patients with an average tumor shrinkage of $35 \%$ (one CR, one $50 \%$ tumor regression, two stable bulky tumors reduced for 25\%). Significant $\mathrm{AE}$ was observed in three patients treated at "most favourable" time-position (in lung and lymph node metastases, and in primary breast cancer) (Fig. 4), while in one among those treated at "least favourable" time-position (in lymph node metastases). Two patients with CR (one from each treatment group) were submitted two months after SBRT-PATHY to surgery, which confirmed pathologic $\mathrm{CR}$ at the level of the partially treated bulky tumor but also regional unirradiated lymph node metastases in both patients. Six out of eight patients were free from progression among which all four which were treated at "most favourable" day. In all these four patients was also achieved the symptom relief while among those treated at "least favourable" day it was observed in three patients. Four patients (two from each treatment group) experienced fatigue grade 1 . No patient reported any late toxicity. 


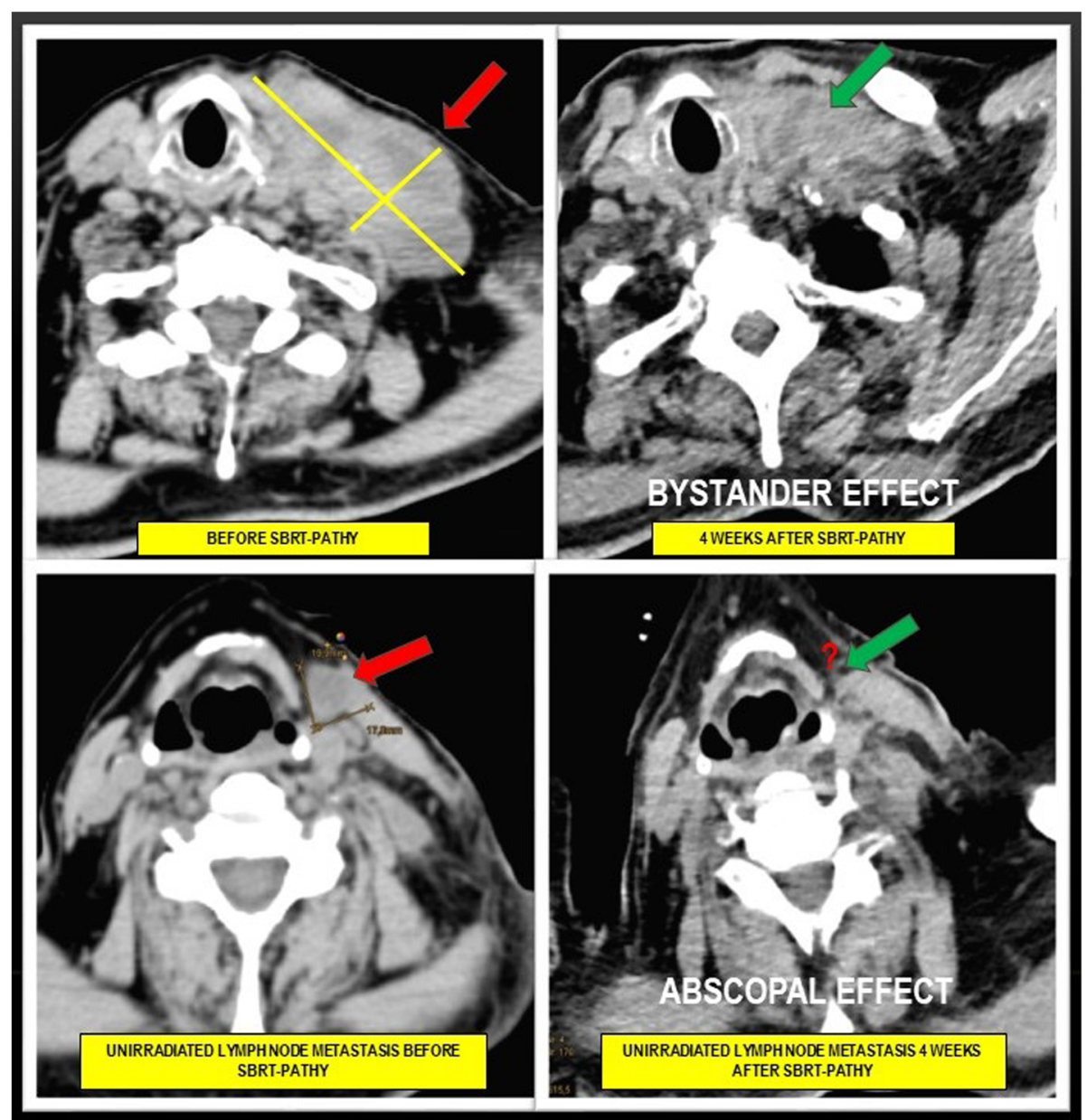

Fig. 4 Induction of the bystander and abscopal effects by time-synchronized immune-guided SBRT-PATHY: Figure shows a very voluminous lymph node metastasis of the left neck in metastatic melanoma patient, with a maximum diameter of $10 \times 7 \mathrm{~cm}$ before SBRTPATHY (red arrow, upper image left), as well as one additional lymph node metastasis with a maximum diameter $2 \times 2 \mathrm{~cm}$ that was not irradiated (red arrow, lower image left). A dramatic regression of the partially treated bulky lesion and also of unirradiated smaller lymph node metastasis was observed 4 weeks later (green arrows, upper and lower images right)

\section{Discussion}

In addition to the partial tumor irradiation, sparing the loco-regional immune tumor microenvironment, the timing of radiotherapy in relation to the different phases of immune response could be the critical "missing link". Recent evidences suggested that timing of therapy may influence clinical outcomes via immune modulation of the underlying immune response-suppression rather than direct tumor effects $[18,19]$. In order to serially map the homeostatic immune response-fluctuations, we used Hs-CRP, LDH and LMR as surrogate of immune system interactions $[15,18,19]$. Since CRP synchronously rises and falls with initiation and termination of the immune response, we determined the start of the cycle as the "most favourable" day of the immune cycle "to release tumor antigen" by SBRT-PATHY, while the first day(s) of CRP fall as the "least favourable" (Fig. 2). This observations were accompanied with clinical outcomes suggesting significant BE/AE with the "most favorable day" approach.

The effectiveness in terms of $\mathrm{BE} / \mathrm{AE}$ response rate of our novel concept could be explained by preserving the pre-existing/endogenous immune signaling in the nonirradiated tumor segment to be modulated by a sufficient threshold of cellular debris/antigen flow by SBRTPATHY-induced cell damage. This could be seen as analogous to "radio-vaccination" event of manipulating the immunologic homeostatic balance of responsiveness and tolerance via endogenous inflammatory signals. In particular, in order to successfully modulate/disturb the homeostatic tumor immune-suppression, the immune system needs to be preserved as a real OAR.

\section{Conclusions}

To our knowledge, this is the first evidence of a prospectively collected time-synchronized immune-guided 
radiotherapy in treating unresectable bulky tumor patients. We believe the right synchronization of radiotherapy with the homeostatically oscillating immune response may improve the probability of inducing $\mathrm{BE} / \mathrm{AE}$. Larger prospective trial on time-synchronized immuneguided SBRT-PATHY is ongoing at our institute.

\section{Acknowledgements}

Authors would like to thanks to Prim. Dr. Raunik W. for the general support in performing the study.

\section{Authors' contributions}

M.A. and B.J. helped with analysis of the results and write the manuscript. All authors read and approved the final manuscript.

\section{Funding}

None.

\section{Availability of data and materials}

All data generated or analysed during this study are included in this published article and its supplementary information files. Any eventual further details on the data related to this study, are available from the corresponding author on reasonable request.

\section{Ethics approval and consent to participate}

All procedures performed in present study were in accordance with the ethical standards. The informed consent was obtained for each patient before any medical act is performed. Present study has been registered by the local ethic committee for Austrian region „Kärnten "in Klagenfurt (AUT), under study number A 37/19.

\section{Consent for publication}

Not applicable.

\section{Competing interests}

Tubin Slavisa M.D. reported on international patent application PCT/EP2019/ 052164 published as WO 2019/162050. The authors reported no other conflicts of interest.

\section{Author details}

'KABEG Klinikum Klagenfurt, Institute of Radiation Oncology, Feschnigstraße 11, 9020 Klagenfurt am Wörthersee, Austria. ${ }^{2}$ Melbourne, Australia. ${ }^{3}$ BiolRC, R\&D Center for Biomedical Research, Kragujevac, SERBIA, Research Institute of Clinical Medicine, 13 Tevdore Mgvdeli St., 0112 Tbilisi, Georgia.

Received: 7 November 2019 Accepted: 20 November 2019

Published online: 04 December 2019

\section{References}

1. Dagoglu N, Karaman S, Caglar HB, et al. Abscopal effect of radiotherapy in the immunotherapy era: systematic review of reported cases. Cureus. 2019; 11(2):e4103.

2. Tubin S, Raunik W. Hunting for abscopal and bystander effects: clinical exploitation of non-targeted effects induced by partial high-single-dose irradiation of the hypoxic tumour segment in oligometastatic patients. Acta Oncol. 2017:56(10):1333-9.

3. Tubin S, Ahmed MM, Gupta S. Radiation and hypoxia-induced non-targeted effects in normoxic and hypoxic conditions in human lung cancer cells. Int J Radiat Biol. 2018;94:199-211.

4. Tubin S, Popper HH, Brcic L. Novel stereotactic body radiation therapy (SBRT)-based partial tumor irradiation targeting hypoxic segment of bulky tumors (SBRT-PATHY): improvement of the radiotherapy outcome by exploiting the bystander and abscopal effects. Radiat Oncol. 2019;14(1):21.

5. Massaccesi M, Dinapoli N, Boldrini L, et al. Re-treatment of recurrent bulky lesions with high single dose partial irradiation targeting the hypoxic tumor segment (PITH): a case series. Int J Radiat Oncol Biol Phys. 2019;105(Suppl 1):E578. Available at https://www.redjournal.org/article/S0360-3016(19)32 087-5/fulltext

6. Tubin S. Partial tumor irradiation exploiting immunomediated effects. tumor microenvironment as a new oar. Radiother Oncol. 2019;133(Suppl 1):S467-8. (abstract 0886). Available at https://www.thegreenjournal.com/article/S01678140(19)31306-4/pdf

7. Tubin S, Khan KM, Salerno G, et al. Mono-institutional phase 2 study of innovative stereotactic body radiotherapy targeting partial tumor hypoxic (SBRT-PATHY) clonogenic cells in unresectable bulky non-small cell lung cancer: profound non-targeted effects by sparing peri-tumoral immune microenvironment. Radiat Oncol. 2019;14:212. https://doi.org/10.1186/ s13014-019-1410-1.

8. Grossman SA, Ellsworth S, Campian J, et al. Survival in patients with severe Lymphopenia following treatment with radiation and chemotherapy for newly diagnosed solid tumors. J Natl Compr Canc Netw. 2015;13(10):1225-31.

9. Balmanoukian A, Ye X, Herman J, et al. The association between treatmentrelated Lymphopenia and survival in newly diagnosed patients with resected adenocarcinoma of the pancreas. Cancer Investig. 2012;30(8):571-6.

10. Quevedo F, Ashdown ML, Suman VJ, et al. Possible therapeutic reversal of immune suppression in patients with metastatic melanoma by timed delivery of temozolomide chemotherapy: a pilot study. J Clin Oncol. 2009; 27(suppl):e20013.

11. Awwad M, North RJ. Sublethal, whole-body ionizing irradiation can be tumor promotive or tumor destructive depending on the stage of development of underlying antitumor immunity. Cancer Immunol Immunother. 1988:26(1):55-60.

12. Beyranvand NE, Marij JP, Welters A, et al. The importance of correctly timing cancer immunotherapy. Expert Opin Biol Ther. 2017;17(1):87-103.

13. Coventry BJ, Ashdown ML, Quinn MA, et al. CRP identifies homeostatic immune oscillations in cancer patients: a potential treatment targeting tool? J Transl Med. 2009;7:102.

14. Leontovich AA, Dronca RS, Suman VJ, et al. Fluctuation of systemic immunity in melanoma and implications for timing of therapy. Front Biosci (Elite Ed). 2012;4:958-75.

15. Leontovich AA, Dronca RS, Nevala WK, et al. Effect of the lymphocyte-tomonocyte ratio on the clinical outcome of chemotherapy administration in advanced melanoma patients. Melanoma Res. 2017:27(1):32-42.

16. Novák B, Tyson JJ. Design principles of biochemical oscillators. Nat Rev Mol Cell Biol. 2008:9(12):981-91.

17. Dutcher JP, Wiernik PH. Deconstructing and Reinventingthe IL-2 Paradigm: can alternate dosing schedules enhance tumor effect? Kidney Cancer J. 2012;11(1):22-7.

18. Schaue D, Micewicz ED, Ratikan JA, et al. Radiation and inflammation. Semin Radiat Oncol. 2015;25(1):4-10.

19. Messenheimer DJ, Jensen SM, Afentoulis ME, et al. Timing of PD-1 blockade is critical to effective combination immunotherapy with anti-OX40. Clin Cancer Res. 2017;23(20):6165-77.

\section{Publisher's Note}

Springer Nature remains neutral with regard to jurisdictional claims in published maps and institutional affiliations.

Ready to submit your research? Choose BMC and benefit from:

- fast, convenient online submission

- thorough peer review by experienced researchers in your field

- rapid publication on acceptance

- support for research data, including large and complex data types

- gold Open Access which fosters wider collaboration and increased citations

- maximum visibility for your research: over $100 \mathrm{M}$ website views per year

At $\mathrm{BMC}$, research is always in progress.

Learn more biomedcentral.com/submissions 